\title{
Flexibles Bodenfördersystem für den Montageprozess
}

\author{
Dipl.-Ing. Jörg Hübler, Prof. Dr.-Ing. Klaus Nendel, Dipl.-Ing. Uwe Dombeck \\ Technische Universität Chemnitz \\ Institut für Fördertechnik und Kunststoffe (IFK) \\ Professur Fördertechnik
}

\begin{abstract}
Bei Montageprozessen werden flexible Transportsysteme, wie Plattenketten-Transfersysteme, zur praxisgerechten Verkettung von manuellen Arbeitsplätzen, automatischen Montage- und Prüfplätzen sowie Werkzeugmaschinen eingesetzt. Verkettungslösungen für manuelle und automatische Arbeitsstationen in Fertigungs- und Montagelinien bilden Staurollenketten- und Gurtförderer. Bei den am Markt verfügbaren Systemen wird ein allseitiger Werkstückzugang bei den einzelnen Montageabfolgen nur bedingt erreicht. In Zusammenarbeit mit der ALTRATEC Montagesysteme $\mathrm{GmbH}$ wurde ein flexibles Bodenfördersystem für den Montageprozess entwickelt, das eine verbesserte Werkstückzugänglichkeit aufweist und ein barrierefreies Arbeiten ermöglicht. Der Stauförderer arbeitet im kontinuierlichen Fließbetrieb, ist voll kurvengängig und kann Nebenstrecken bedienen. Ein Power \& Free System bildet die Grundlage einer energieeffizienten Auslegung der Anlage und ermöglicht ein Arbeiten auch an wechselnden Arbeitsplätzen mit Taktbetrieb. Mit einem ausführlichen Test des Funktionsmusters konnten alle relevanten Betriebszustände verifiziert werden. Viele Anforderungen von selbstfahrenden Werkstück-Transportsystemen können mit dem neuen System bereits erfüllt werden.
\end{abstract}

\section{$1 \quad$ Einleitung}

Höhere Produktvielfalt, ständig steigender Kostendruck und immer kürzere Innovationszyklen bei gleich bleibend guter Produktqualität im Maschinen- und Fahrzeugbau sowie Elektro- und Haushaltgeräteindustrie erfordern eine immer größere Flexibilität in den Fertigungs- und Montageprozessen. Konventionelle Transfersyteme stoßen dabei oft an ihre Grenzen der Fertigungsstruktur wie Zugänglichkeit, Flexibilität und Handling. Eine Neuanordnung, Anpassung und Modifizierung bestehender Transportsysteme können oftmals nur bedingt den gestiegenen Anforderungen standhalten. Eine vorhandene Anlage umzubauen, um diese an den geänderten Bedingungen anzupassen, ist in den meisten Fällen ökonomisch nicht sinnvoll.

Flexible Fertigungs- und Montagelinien entstehen durch eine miteinander kombinierte Verkettung von Montageplätzen mit Transportsystemen. Die als Schnittstelle zwischen Montage- und Bearbeitungsstationen eingesetzte Transfertechnik hat einen bedeutenden Anteil an der Qualität und Zuverlässigkeit der Verkettung in Montagestrecken. Moderne Transfertechnik realisiert aber nicht nur den eigentlichen Transport, sondern muss in Verbindung mit der Steuerung 
gleichzeitig die exakte Zuführung, Taktung und Positionierung der Bauteile für Fertigungs-, Verarbeitungs-, Montage- oder Überwachungsprozesse übernehmen. Eine weitere wichtige Eigenschaft dieser Transportsysteme ist ihre flexible Anpassung an spezielle Einsatzbedingungen.

Einen neuen Ansatz bietet dabei die Entwicklung eines alternativen Werkstückträgertransfersystems, welches als flexibel und modular aufgebautes kurvengängiges Bodenfördersystem nahezu unabhängig von den baulichen Bedingungen der Produktionsstätte eingesetzt werden kann.

\section{Entwicklung eines neuartigen Montagetransfersystems}

Die bodengebundene Fördertechnik wird in den meisten Fällen individuell an den spezifischen Anwendungen in Fertigungs- und Montageprozessen angepasst. Folgende grundlegende Bodenfördersysteme werden dabei unterschieden:

- Skidsysteme

- Schubskidsysteme

- Fahrerlose Transportsysteme (FTS)

- Elektropalettenbahnen (EPB)

- Automatische Warenverteilsysteme

- Rollenbahnsysteme

- Bandförderanlagen

- Unterflurförderer

- Kettenförderer

Unter Beachtung der in [Lot06] genannten Einflussfaktoren auf die Verfügbarkeit von Montagesystemen wurde ein neues, innovatives Bodenfördersystem entwickelt, das in den Kategorien Unterflurförderer und Kettenförderer einzuordnen ist.

Die Innovation dieses Systems liegt in der vollständigen Begehbarkeit und der damit verbundenen großzügigen Werkstückzugänglichkeit sowie einer Power \& Free Lösung für die Werkstückträgerwagen. Anders als bei den bekannten, im Hallenboden integrierten Bodenförderern, baut das neue System direkt auf dem Hallenboden auf. Die Werker können sich frei auf einer extrem niedrig bauenden Arbeitsplattform bewegen. Richtungsgeführte, auf Laufrollen montierte Werkstückträgertransportwagen laufen formschlüssig im Schleppketteneingriff auf einer Bodenplatte kurz unterhalb der Arbeitsplattform. Mittels eines mechanischen Power \& Free Systems können einzelne Werkstückträgerwagen über Nebenstrecken ein- bzw. ausgeschleust und kraftfrei gestaut werden. Eine Automatisierung von aufwendigen Montagearbeitsplätzen mit speziellen Werkstückgeometrien konnte somit realisiert werden. 


\subsection{Systemanforderungen}

Die allgemeinen Anforderungen an moderne Werkstück-Transportsysteme in Fertigungsbereichen sind vielfältig und müssen sich den gegebenen Strukturen anpassen. Neben der Zugänglichkeit zum Werkstück, der Ergonomie, Sicherheit und Umweltverträglichkeit ist dies insbesondere die Flexibilität des Systems [VDI08].

Weitere anwendungsbezogene Anforderungen an das System:

- Herausnehmen bzw. Einfügen der Wagen

- Bedienen von Nebenstrecken

- kontinuierlicher Fließbetrieb

- Taktbetrieb durch Power \& Free System an den Haltepunkten

- Puffermöglichkeit durch Staubetrieb

- modularer Aufbau

- Positionierung Werkstückträger für manuelle bzw. automatische Arbeitsstationen Abmessungen, Massen, Verstellmöglichkeiten und Fahrgeschwindigkeiten wurden vorab genau definiert.

\subsection{Funktionsprinzip}

Bei der Entwicklung von Lösungsvarianten stand klar die Erfüllung der Forderungen aus dem Pflichtenheft im Vordergrund. Eine ausführliche Recherche der am Markt befindlichen flurgebundenen Schleppkettenfördersysteme bildete ebenso eine entscheidende Grundlage. Daraus abgeleitete Lösungsvarianten wurden für das Gesamtsystem und den Einzelkomponenten entwickelt.

Das in den Abbildung 1 dargestellte Beispiel eines Anlagenlayouts des Bodenfördersystems besteht aus drei unabhängigen Schleppkettensystemen, die mit der gleichen Geschwindigkeit laufen. Es werden sowohl innen als auch außen geschleppte Kurven realisiert. D. h. der Werkstückträgerwagen besitzt beidseitig Mitnehmer, die wahlweise den Antrieb von links oder rechts bewirken. Die gestrichelten Linien stellen schematisch den Kettenverlauf dar. An den Überlappungsstellen der Schleppkettensysteme erfolgt eine mechanische Kopplung der Werkstückträgertransportwagen. An diesen Stellen kann eine Übergabe an Nebenstrecken erfolgen. Die Transportwagen sind mit einem mechanischen Power \& Free System ausgerüstet, das beim Auffahren auf einem stehenden Wagen oder einen zugeschalteten Stoppmechanismus den Formschluss mit der Schleppkette aufhebt, so dass keine Staukräfte entstehen. Geführt werden die Transportwagen in einer Nut in der oberen Bodenplatte. Damit wird eine definierte Position der Wagen gewährleistet. 


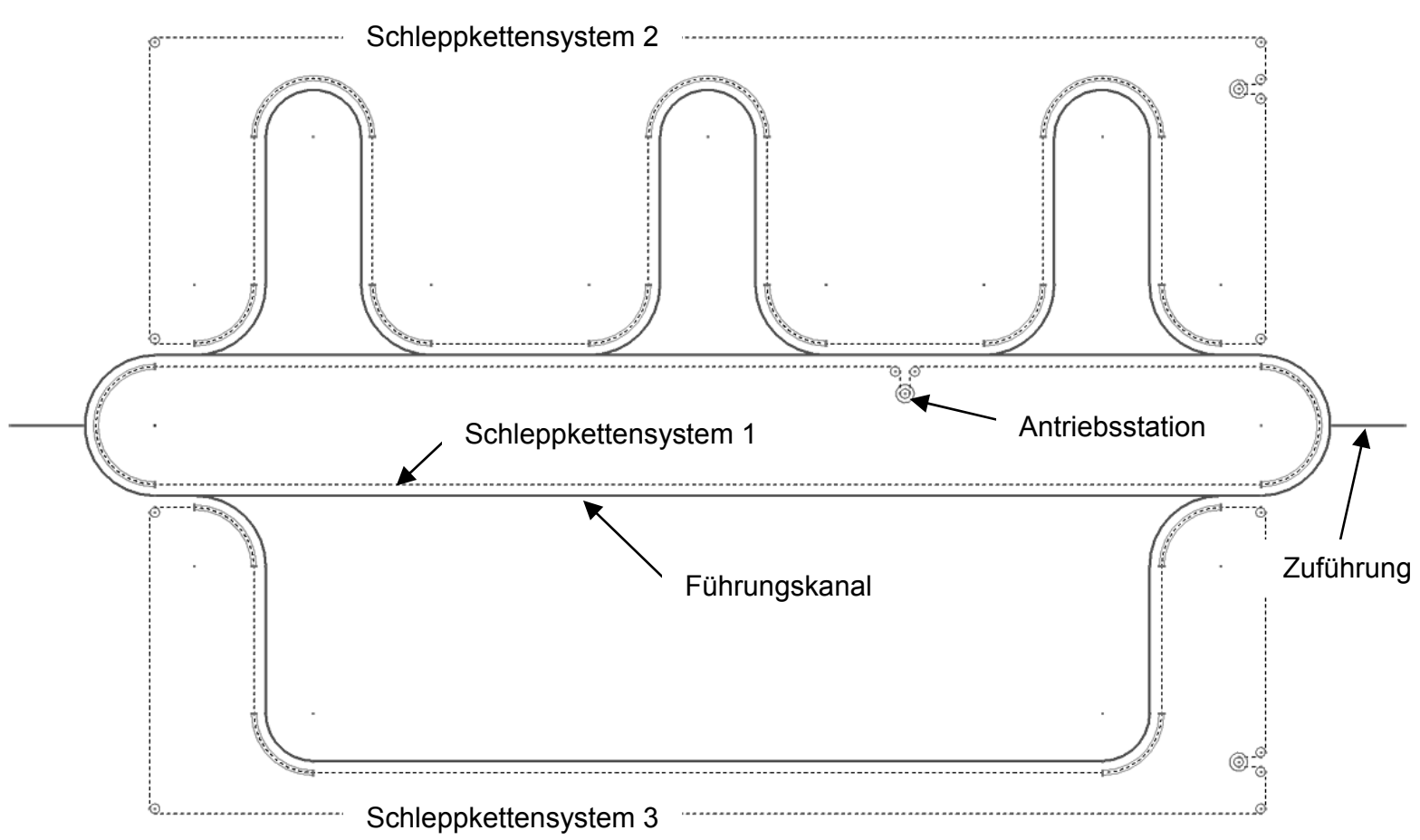

Abbildung 1: Beispiel Anlagenlayout

\subsection{Bodensystem in Sandwichbauweise}

Bekannte Unterflurförderer werden mit hohem bautechnischem Aufwand in den Hallenboden integriert. Bodengeführte Kettenfördersysteme sind auf dem Boden installiert, verhindern aber durch inren Streckenaufbau ein freies Bewegen der Werker bei Montagearbeiten. Um diese Probleme zu umgehen wurde ein Bodensystem entwickelt, das auf dem vorhandenen Hallenboden aufbaut und dazu ein barrierefreies Begehen ermöglicht. Als Plattensystem wurden Sperrholzplatten verwendet, deren Oberflächen mit einer hoch abriebfesten Filmbeschichtung versehen sind. Darüber hinaus sind die Platten gegen Feuchtigkeit und handelsüblichen Chemikalien beständig. Die mechanische Bearbeitung erfolgt mit Holzbearbeitungswerkzeugen. Die Platten sind einseitig mit einer Gitterstruktur versehen, die hervorragende rutschhemmende Eigenschaften aufweist. Der Bodenaufbau erfolgt nach dem Sandwichprinzip. Zur Abstützung der Platten werden Aluminiumprofile mit den Platten verschraubt. In dem entstandenen Zwischenboden läuft das Schleppkettensystem mit den entsprechenden Werkstückträgerwagen, Abbildung 2. Untereinander sind die Platten durch ein Nut-Federsystem fixiert. Die Zuführung der Versorgungsmedien erfolgt direkt von oben. 


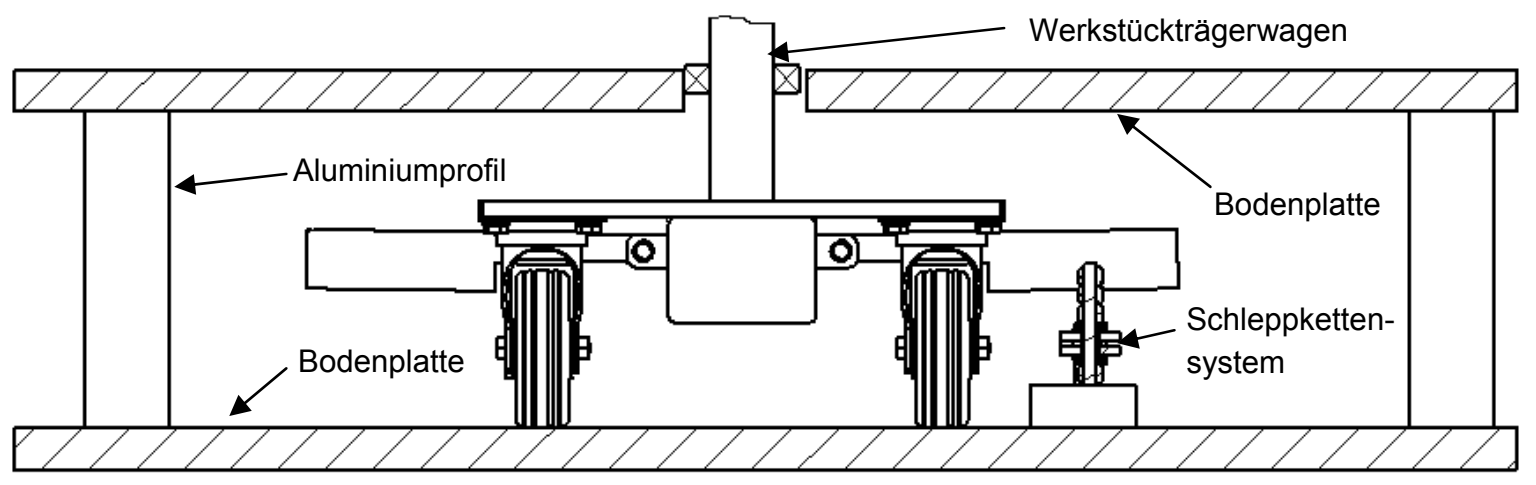

Abbildung 2: Bodenaufbau

\subsection{Werkstückträgerwagen}

Der Werkstückträgerwagen besteht aus einem nicht selbst fahrenden Chassis, das mit Gelenkrollen versehen ist. Darauf ist eine in der Höhe verstellbare, um die ZAchse $360^{\circ}$ drehbare und um $+/-90^{\circ}$ schwenkbare Werkstückträgeraufnahme aufgebaut, Abbildung 3.

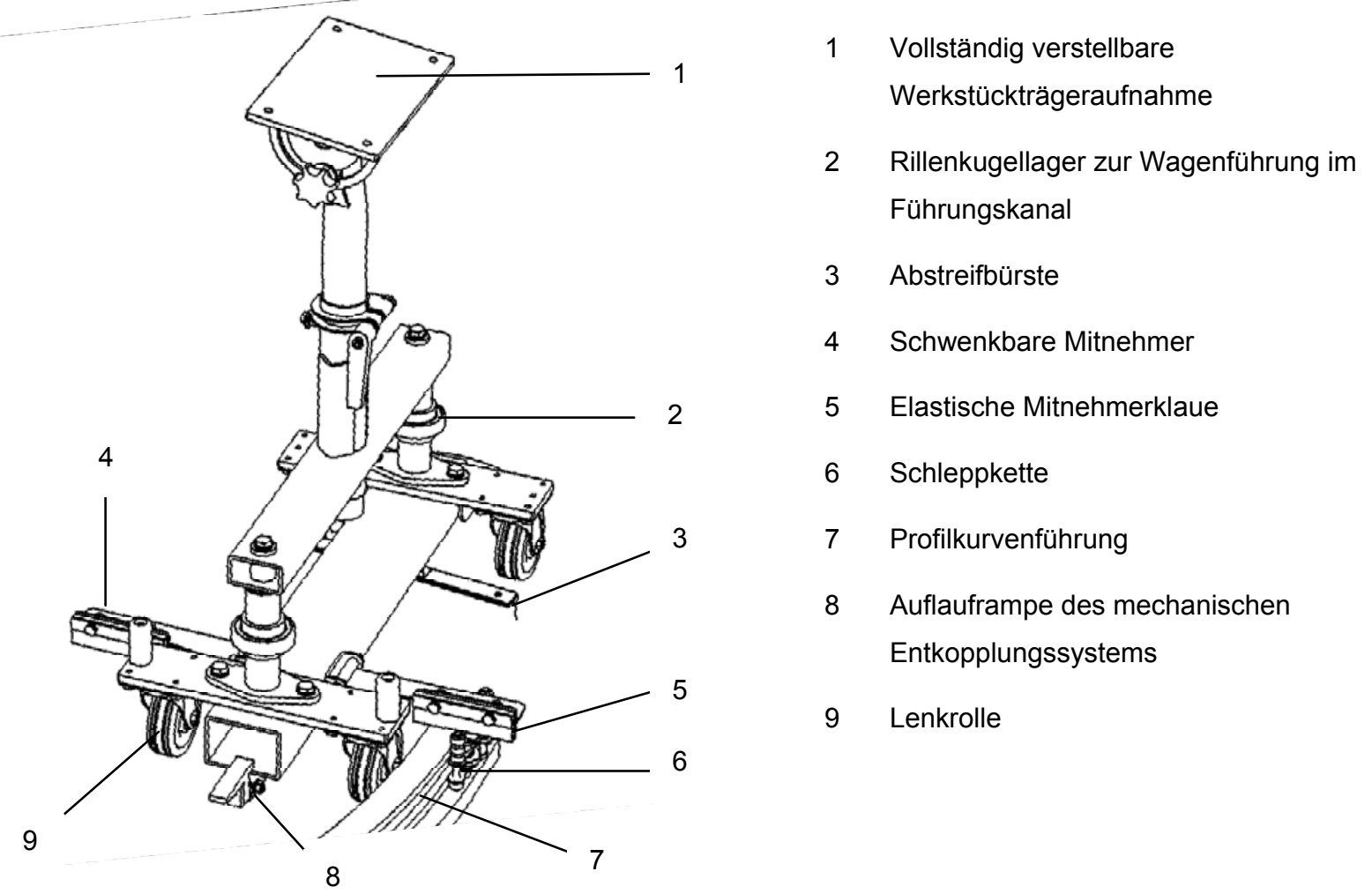

Abbildung 3: Aufbau Werkstückträgerwagen

Die Führung des Werkzeugträgerwagens erfolgt über den im vorderen und hinteren Bereich des Wagens angeordneten Rillenkugellagern, die sich am Führungskanal der oberen Bodenplatte abstützen. 


\subsection{Beschreibung der Wirkprinzipien}

\subsubsection{Schleppkettensystem}

Die Entwicklung des Schleppkettensystems erfolgte zusammen mit dem Power \& Free System der Transportwagen und den Gleitführungen. Basierend auf einer Staurollenkette $3 / 4$ "' in einer Sonderausführung mit einseitiger Rollenanordnung 3-1, wurde eine Spezialschleppkette entwickelt, Abbildung 4. Die verlängerten Bolzen dienen als Mitnehmer der Werkstückträgerwagen und die zwischen den Kettengliedern angeordneten Rollen stützen die Schleppkette in den Kurvenprofilen so ab, dass Rollreibung zwischen Kette und Profil entsteht.

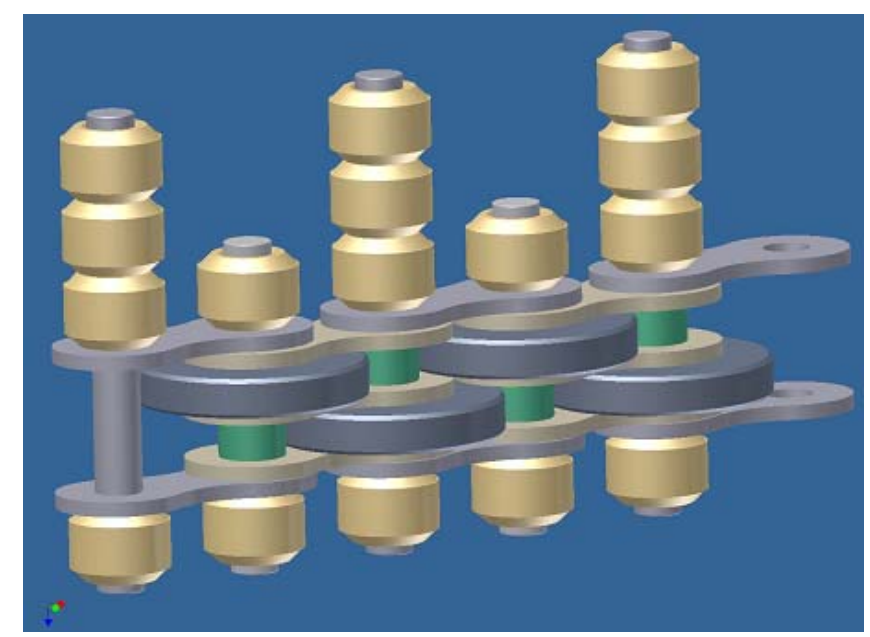

Abbildung 4: Spezialschleppkette

Die vertikal angeordnete Schleppkette wird in den Kurven entweder mittels Gleitschienen oder über polygonförmig angeordnete Kettenräder geführt, Abbildung 5 und 6 .

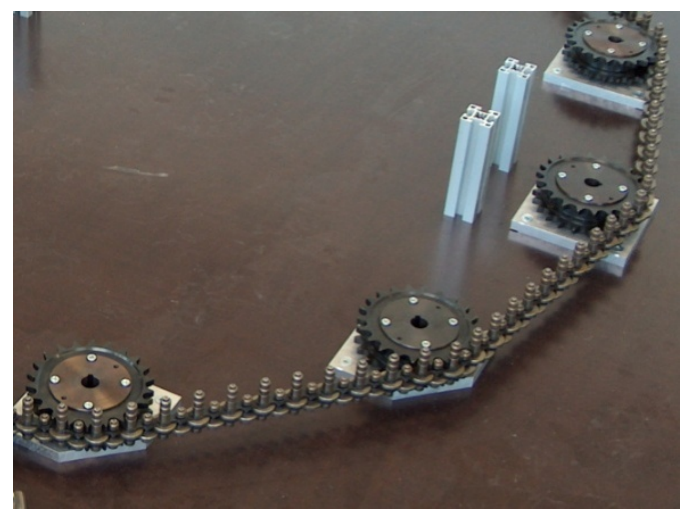

Abbildung 5: Kettenradkurve

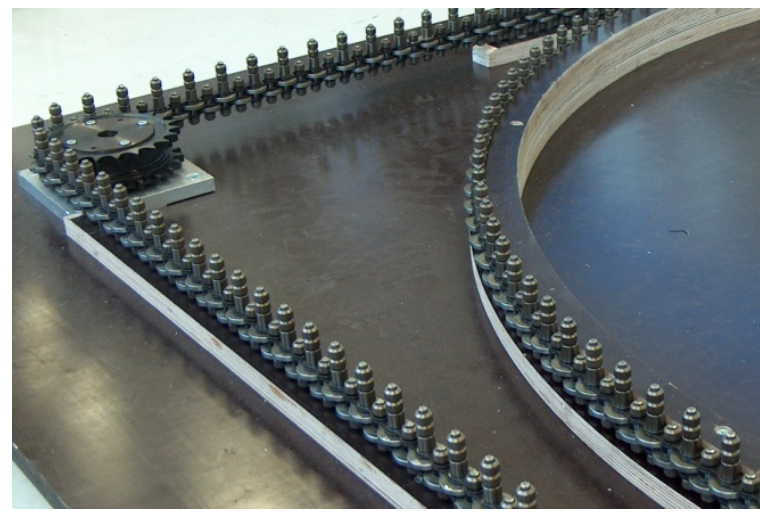

Abbildung 6: Gleitleiste und Gleitkurve

Bei den Kettenradkurven entstehen nur geringe Verluste auf Grund der Wälzreibung zwischen Schleppkette und den Kettenrädern.

Bei der Profilkurve stützt sich die Schleppkette an der Kettenkontur angepasste Holzführung radial ab. Auf den geraden Kettenabschnitten dienen einfache 
Sperrholzgleitleisten als Unterstützung, um ein Durchhängen der Schleppkette zu vermeiden. Als Antrieb wurden Standard-Kettenradantriebe mit federbelastetem Spanner verwendet.

\subsubsection{Wagenmitnahme}

Horizontal schwenkbare Klauen dienen zur Wagenmitnahme. Die Klauen greifen direkt zwischen den verlängerten Kettenbolzen der Schleppkette ein.

Bei der Wagenübergabe an Nebenstrecken wechselt der Klaueneingriff Mitnehmer / Schleppkette von einem zum anderen Schleppkettensystem. Auf der Bodenplatte befestigte Einlauframpen steuern den Eingriff der Mitnehmer, Abbildung 7. Eine pneumatisch oder elektrisch geschaltete Weiche leitet dann den Werkstückträgerwagen auf die Nebenstrecke. Beim Einschleusen ist durch die Zusammenführung von zwei Linien keine Schaltweiche erforderlich.

Die Mitnehmerklauen bestehen aus Polyurethan-Plattenmaterial. Dieser Werkstoff zeichnet sich durch hohe Elastizität und extremer Wiederstandfähigkeit gegenüber mechanischen Beanspruchungen aus. Bei Blockade der Fahrbahn werden die Mitnehmer soweit elastisch verformt, bis es zum Überspringen der Mitnehmerklauen über den Kettenbolzen kommt und der Wagen stoppt.

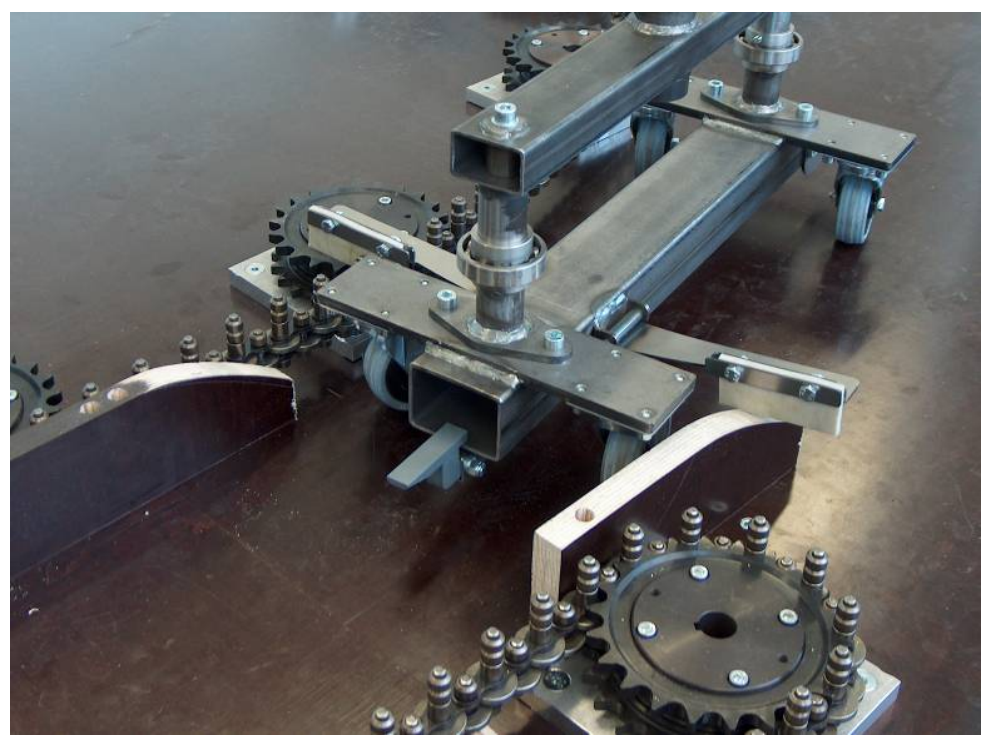

Abbildung 7: Einlauframpen

\subsubsection{Power \& Free System}

Traditionelle Power \& Free Anlagen basieren auf einer Mitnehmer/Klinkenkonstruktion, die eine formschlüssige Verbindung zur Powerkette herstellt. Dabei sind die Klinken so ausgeführt, dass beim Auflaufen auf einer Stapelkufe die Verbindung zum Mitnehmer gelöst wird [Pie10].

In Anlehnung an dieses System wird beim Auffahren eines Werkstückträgerwagens auf einen stehenden eine Auflauframpe am vorderen Teil des Wagens betätigt, die mechanisch gleichzeitig beide Mitnehmer nach oben schwenkt und somit den 
Formschluss mit der Schleppkette aufhebt, Abbildung 8. Bewegt sich der vordere Wagen weiter, kehrt sich dieser Vorgang um und der Schleppketteneingriff ist wiederhergestellt.

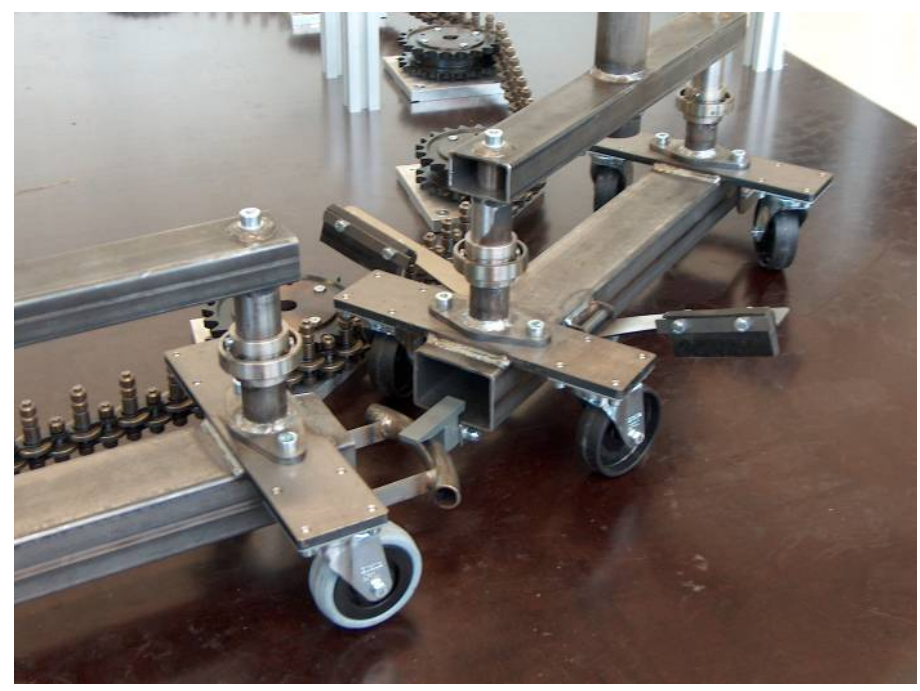

Abbildung 8: Power \& Free System

Um den Wagen am Montagearbeitsplatz zu stoppen, wird abweichend davon ein schaltbarer Ausheber verwendet, der den Eingriff nur eines Mitnehmers löst und den Wagen stoppt.

\section{Funktionsmuster}

\subsection{Versuchsaufbau}

Die Abmessungen des getesteten Funktionsmusters betrugen $3 \times 3 \mathrm{~m}$. Dieser Platz war ausreichend, um alle relevanten Funktionen und Komponenten des Antriebssystems, des Bodenaufbaus und der Werkstückträgerwagen im Maßstab 1:1 zu testen, Abbildung 9. Zum Einsatz kamen zwei Schleppkettensysteme mit jeweils von Gleitleisten geführten Kurve und einer Kettenradkurve. Somit konnte pro Wagenumlauf zwei Übergabestellen realisiert werden.

Dieser Versuchsaufbau diente zum Einstellen der Funktionsfähigkeit des Fördersystems und zum Test aller umgesetzten Funktionselemente. Ein anschließender Dauerlauf diente zur Nachweisführung und zum Aufzeigen von Schwachstellen. 


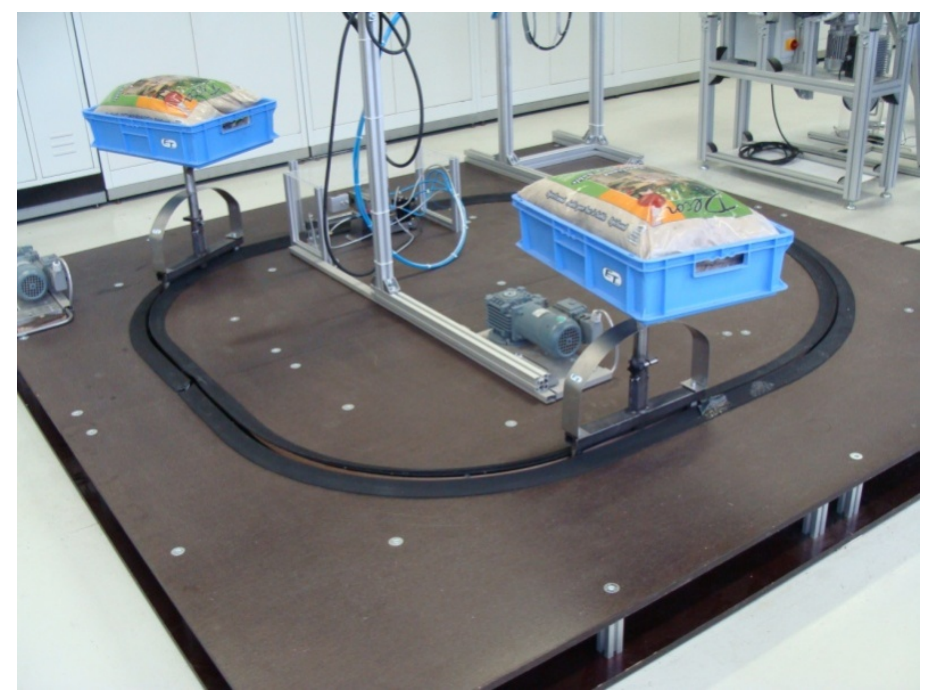

Abbildung 9: Aufbau Funktionsmuster

\subsection{Versuchsdurchführung}

Zwei Werkstückträgerwagen laufen kontinuierlich mit einer Geschwindigkeit von $6 \mathrm{~m} / \mathrm{min}$ um. Die Wagen sind jeweils mit $50 \mathrm{~kg}$ beladen. In diesem Fall wurden $2 \times 25 \mathrm{~kg}$ in Folie eingeschweißte Sandsäcke als Dummygewichte verwendet. Diese liegen in einer auf dem Werkstückträger fest montierten Kunststoffbox. Die Höhe der Testmasse wurde auf die max. geforderten $900 \mathrm{~mm}$ eingestellt. Pro Umlauf wurde jeder Wagen mit der Fixierstation für $3 \mathrm{sec}$ automatisch gestoppt und mit dem Winkelgreifer fixiert, Abbildung 10. Sensoren erkennen dabei die Position des Wagens und senden ein Signal an einem Pneumatikzylinder, der die Mitnehmerklaue aus dem Ketteneingriff löst. Eine Umlaufstrecke betrug dabei $7252 \mathrm{~mm}$.

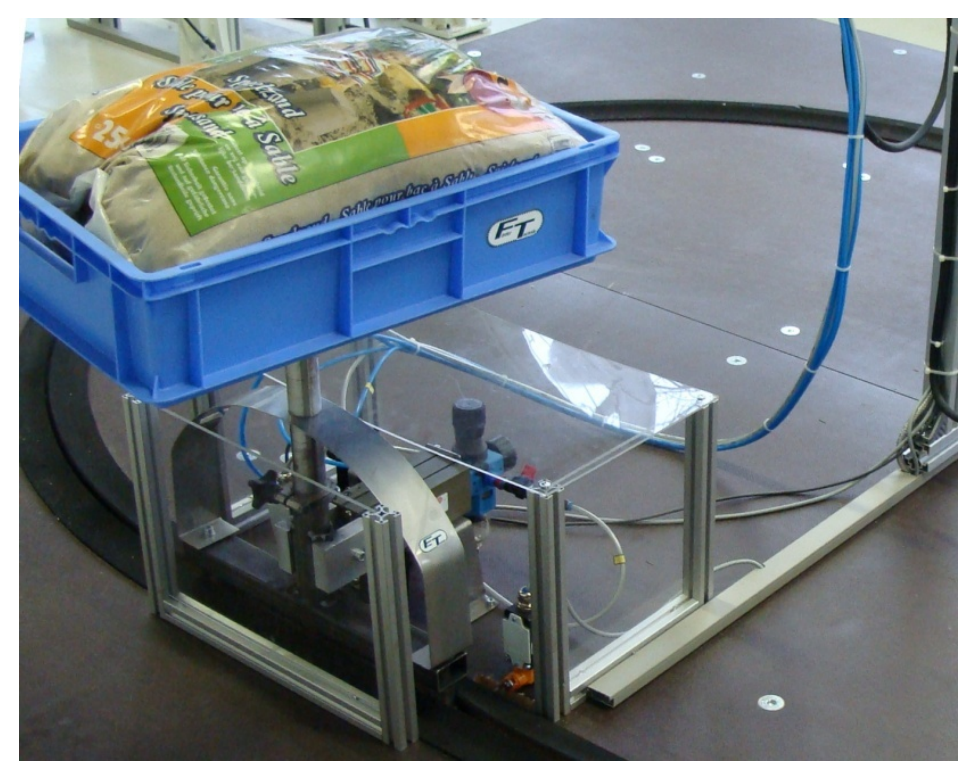

Abbildung 10: Fixierstation 


\subsection{Ergebnisse}

Der Dauerlauf wurde nach $2724 \mathrm{~h}$ beendet. Dieser Zeitraum entspricht etwa im industriellen Einsatz $1 \mathrm{Jahr}$ im Einschichtbetrieb. Dabei legte jeder Werkstückträgerwagen $900 \mathrm{~km}$ zurück und die Fixierstation absolvierte in dieser Zeit 60.000 Schaltzyklen. Danach wurde das gesamte Funktionsmuster zerlegt und alle Wirkpaarungen einer Verschleißbeurteilung unterzogen. Nennenswerter Verschleiß konnte nur an der Oberfläche der unteren Bodenplatten im Bereich der Wagenfahrspur festgestellt werden, Abbildung 11. Bei den verwendeten Gleitleisten konnte minimaler Verschleiß nur im Einlaufbereich festgestellt werden.

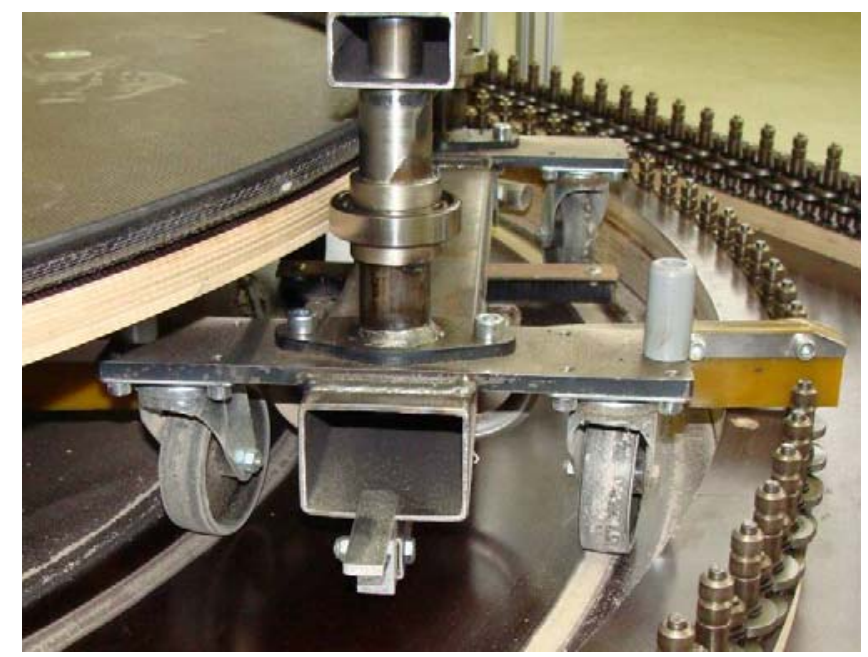

Abbildung 11: Bodenplattenverschleiß in der Fahrspur

\section{$4 \quad$ Energieeffizienz / Dimensionierung}

Aus energetischer Sicht ist es sinnvoll, nur so viele Antriebe einzusetzen wie notwendig. Folgende Faktoren sind dabei zu betrachten. Der Fahrwiderstand der Werkstückträgerwagen berechnet sich aus der Summe von Rollwiderstand und Lagerreibung, Abbildung 12.

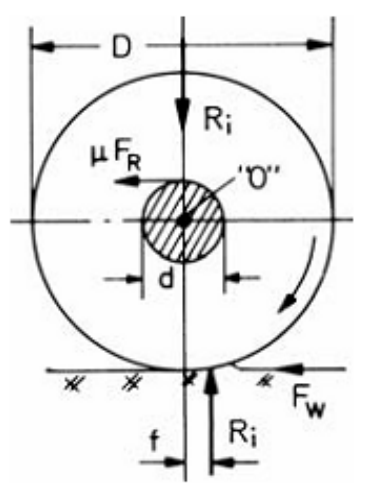

$$
F_{w}=\frac{R_{1}}{D}(2 f+\mu d)_{\#}
$$

Abbildung 12: Fahrwiderstand [Mar08]

Für die Gleitreibung zwischen Schleppkette und Gleitführungen ist die Gewichtskraft und der Reibwert ausschlaggebend, Abbildung 13. 


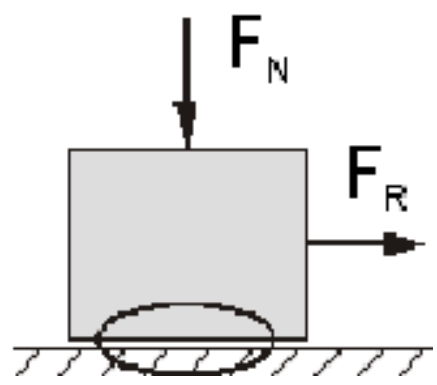

Abbildung 13: Gleitreibung

Für eine Dimensionierung des Systems spielen die Gelenkreibung der Schleppketten, Rollreibung der Wagenführungen, Rollreibung an den Gleitkurven, Wälzreibung an der Kettenrädern und Verformungswiderstände der elastischen Mitnahmeklauen beim ungewollten Blockieren nur eine untergeordnete Rolle und müssen über einen entsprechenden Betriebsfaktor berücksichtigt werden.

\section{$5 \quad$ Zusammenfassung und Ausblick}

Es wurde ein neuartiges, flexibles Flurfördersystem für den Montageprozess entwickelt, wobei der Aufbau direkt auf dem Boden erfolgt und in seiner gesamten Ausdehnung vollständig begehbar ist. Durch den modularen Aufbau kann das Fördersystem sehr flexibel den jeweiligen Anforderungen angepasst werden.

Dieses System besteht aus kontinuierlich umlaufenden Schleppkettensystemen und Werkstückträgerwagen, die sich innerhalb eines niedrig bauenden Plattensystems in Sandwichbauweise befinden. Die Werkstückträgerwagen werden formschlüssig und kontinuierlich von der Schleppkette mitgenommen. Jeder Wagen verfügt über jeweils einen rechts und links angeordneten schwenkbaren Mitnehmer, der mit einer elastischen Klaue ausgerüstet ist. Neben der allseitigen Zugänglichkeit der Werkstücke können die Wagen Nebenstrecken bedienen, kraftflussfrei gestaut und an den Montagearbeitsplätzen festgesetzt werden. Nach erfolgreicher Testphase des Funktionsmusters besteht nun die Möglichkeit, das Fördersystem zu überarbeiten, am Markt vorzustellen und kundenspezifischen Anforderungen anzupassen.

\section{Technische Daten:}

- Werkstückgewicht bis $50 \mathrm{~kg}$

- Werkstückträger bis zu $900 \mathrm{~mm}$ höhenverstellbar und allseitig zugänglich; horizontal $+/-90^{\circ}$ schwenkbar und vertikal $360^{\circ}$ stufenlos einstellbar

- Geschwindigkeiten bis max. $6 \mathrm{~m} / \mathrm{min}$

- Herausnehmen bzw. Einfügen von Wagen

- Kontinuierlicher Fließbetrieb

- Taktbetrieb durch Power \& Free System an den Haltepunkten

- Puffermöglichkeit bei Staubetrieb oder Ausschleusen auf Nebenstrecken

- Sicherheits-Stopp-System der Werkstückträgerwagen 


\section{Literatur}

[VDI08] VDI 3640: Selbstfahrendes Werkstück-Transportsystem, Berlin: BeuthVerlag, 2008

[Mar08] Martin, H.; Römisch, P.; Weidlich, A.: Materialflusstechnik, 9., verbesserte und aktualisierte Auflage, Wiesbaden: Vieweg-Verlag, 2008

[Lot06] Lotter, B.; Wiendahl, H.-P.: Montage in der industriellen Produktion, Berlin Heidelberg: Springer-Verlag, 2006

[Pie10] Pietschmann, J.: Industrielle Pulverbeschichtung, 3.,überarbeitete Auflage, Wiesbaden: Vieweg+Teubner, 2010 